\title{
Ureteropelvic junction obstruction and renal cell carcinoma in a patient with solitary functioning kidney
}

\author{
Young Beom Jeong, MD;' Oh Seok Ko, MD;1 Hyung Sub Park, MD;' Jai Seong Cha, MD;l Seung Chol Park, \\ $M D,{ }^{3}$ Hyung Jin Kim, MD;1 Jong Kwan Park, MD;' Yu Seob Shin, MD'
}

\begin{abstract}
'Department of Urology, Chonbuk National University Medical School, and Research Institute of Clinical Medicine of Chonbuk National University-Biomedical Research Institute of Chonbuk National University Hospital, Jeonju , Korea; ${ }^{2}$ Department of Surgery, Seoul National University Bundang Hospital, Seoul National University College of Medicine, Gyeonggi, Korea; ${ }^{3}$ Department of Urology, Wonkwang University School of Medicine, Institute of Wonkwang Medical Science, Iksan, Korea; Dr. Jong Kwan Park and Dr. Yu Seob Shin contributed equally to this work.
\end{abstract}

Cite as: Can Urol Assoc J 2016;10(3-4):E114-6. htrp://dx.doi.org/10.5489/cuai.3002

\section{Abstract}

We present a case of ureteropelvic junction obstruction (UPJO) and renal cell carcinoma (RCC) in a solitary functioning kidney (SFK), managed by robot-assisted dismembered pyeloplasty with partial nephrectomy in a single stage. To our best knowledge, we report the first case of UPJO with RCC in a congenital SFK.

\section{Introduction}

Management of ureteropelvic junction obstruction (UPJO) and renal cell carcinoma (RCC) in a solitary functioning kidney (SFK) can be challenging. Sophisticated correction of the UPJO and resection of the RCC with nephron-sparing were urgently needed to preserve renal function in a SFK. To our best knowledge, UPJO with RCC in a SFK has never been reported in the literature. Furthermore, in this case, UPJO with RCC in a SFK was successfully managed by a one-stage operation with preservation of renal function.

\section{Case report}

A 64-year-old man visited a local clinic for a routine medical checkup. The patient had no specific prior medical and family history. He had no specific symptoms on physical examination and his blood pressure was normal. Hydronephrosis on the left kidney and non-visualization of the right kidney was incidentally detected by ultrasonography (US). Thereafter, he was referred to our hospital for further evaluation.

Computed tomography showed a UPJO and a $2.8 \mathrm{~cm}$ hypervascular renal mass (HRM) in the mid-pole of the left SFK. The serum creatinine $(\mathrm{Cr})$ level was $1.88 \mathrm{mg} / \mathrm{dL}$ and glomerular filtration rate (GFR) was $36.9 \mathrm{ml} / \mathrm{min}$. Magnetic resonance imaging (MRI) showed a $2.8 \mathrm{~cm}$ left HRM with
UPJO (Fig. 1). Renal scan showed a nonfunctioning right kidney and decreased uptake with markedly delayed excretory function in the left kidney. Correction of the obstructive uropathy and resection of the HRM with nephron-sparing surgery were both important to our patient, due to the presence of a SFK. Thus, we performed concomitant robot-assisted laparoscopic pyeloplasty with partial nephrectomy in a single stage. The segmental renal artery of the HRM was selectively clamped to reduce ischemic damage. Ischemic time was 25 minutes. Pathologic evaluation revealed a clear cell RCC, Fuhrman nuclear grade 2/4 (stage: T1aNOM0) (Fig. 2). He recovered from the operation without complications and at three months post-surgery, serum $\mathrm{Cr}$ level and GFR were $1.42 \mathrm{mg} / \mathrm{dL}$ and $51.8 \mathrm{ml} / \mathrm{min}$, respectively. At nine months post-surgery, serum $\mathrm{Cr}$ level and GFR were 1.17 $\mathrm{mg} / \mathrm{dL}$ and $65.5 \mathrm{ml} / \mathrm{min}$, respectively.

\section{Discussion}

A SFK may be associated with urological abnormalities, the most common anomalies being vesicoureteral reflux and hydronephrosis. ${ }^{1,2}$ True renal agenesis results from either failure of formation of the ureteric bud or absence of an interaction with the metanephric mesenchyme. ${ }^{3}$ In our patient, the right kidney and ureter were absent and SFK was a result of true renal agenesis.

The critical decision to be made in dealing with suspected UPJO is whether the radiologic findings correlate with the physiologic picture. ${ }^{4}$ In our patient, UPJO with severe hydronephrosis was detected in a SFK and renal scan revealed decreased uptake with markedly delayed excretory function in the left kidney. Therefore, prompt resolution of the obstructive nephropathy was essential in this case. The gold standard treatment for managing UPJO has been dismembered pyeloplasty over decades. After the first report of laparoscopic pyeloplasty by Schuessler in 1993, the laparoscopic approach has been widely accepted. ${ }^{5}$ Recently, a robotic-assisted approach has become popular. Regardless 


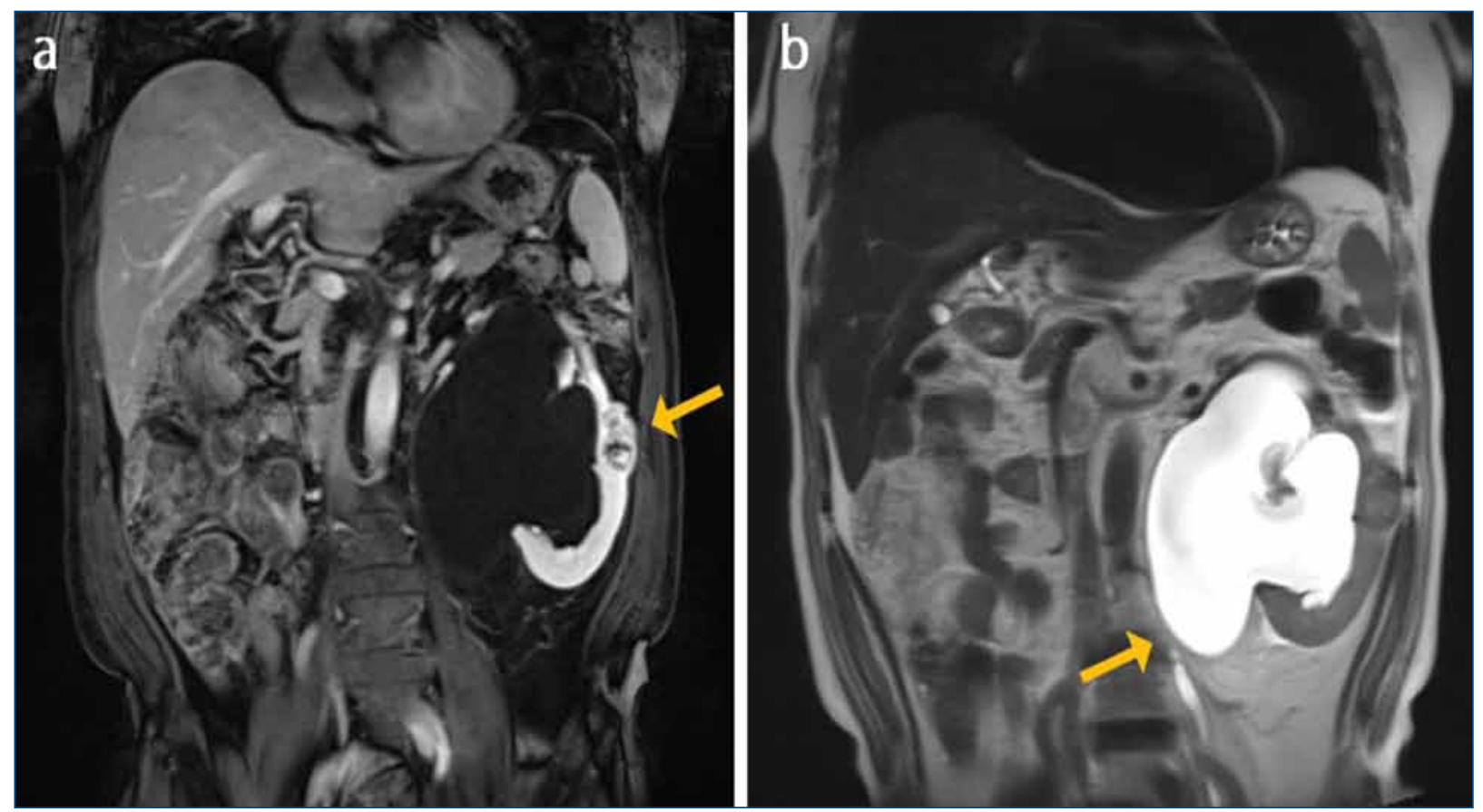

Fig. 1. Magnetic resonance imaging shows a $2.8 \mathrm{~cm}$ hypervascular renal mass (HRM) and ureteropelvic junction obstruction (UPJO) in a left solitary functioning kidney (SFK). (a) T1 coronal image shows a HRM (arrow) in the mid-pole of the left SFK; (b) T2 coronal image shows severe hydronephrosis combined with renal parenchymal thinning due to UPJO (arrow) in the left SFK.

of the approach, the success rate of dismembered pyeloplasty for UPJO is reported to be $>90 \%{ }^{6}$

In our patient, a $2.8 \mathrm{~cm}$ HRM was located in the mid-pole of the SFK with UPJO. The HRM was suspected to be a RCC on imaging studies. Resection of the HRM with nephronsparing was needed to treat the cancer with preservation of renal function. For localized HRM, partial nephrectomy is widely accepted as a standard therapy. Additionally, roboticassisted partial nephrectomy has recently become an alternative to standard laparoscopy, with the advantages of more technologically advanced instrumentation, 7 providing good surgical vision by three-dimension, and accurate mobility for suturing.

The main dilemma was to decide which disease (UPJO or HRM) to treat first in a SFK. We thought that correction of the obstructive uropathy and resection of the HRM with nephron-sparing surgery would both be important to our patient. Thus, we decided to perform concomitant robotassisted laparoscopic pyeloplasty with partial nephrectomy in a single stage. Furthermore, selective clamping of the segmental renal artery was performed to reduce ischemic damage ${ }^{8}$ which may not be of great concern in normal patients, but may lead to postoperative renal function deterioration and need for hemodialysis in patients with a SFK.

In conclusion, UPJO with RCC in a SFK was successfully managed by a one-stage operation with preservation of renal function.
Competing interests: The authors declare no competing financial or personal interests.

This paper has been peer-reviewed.

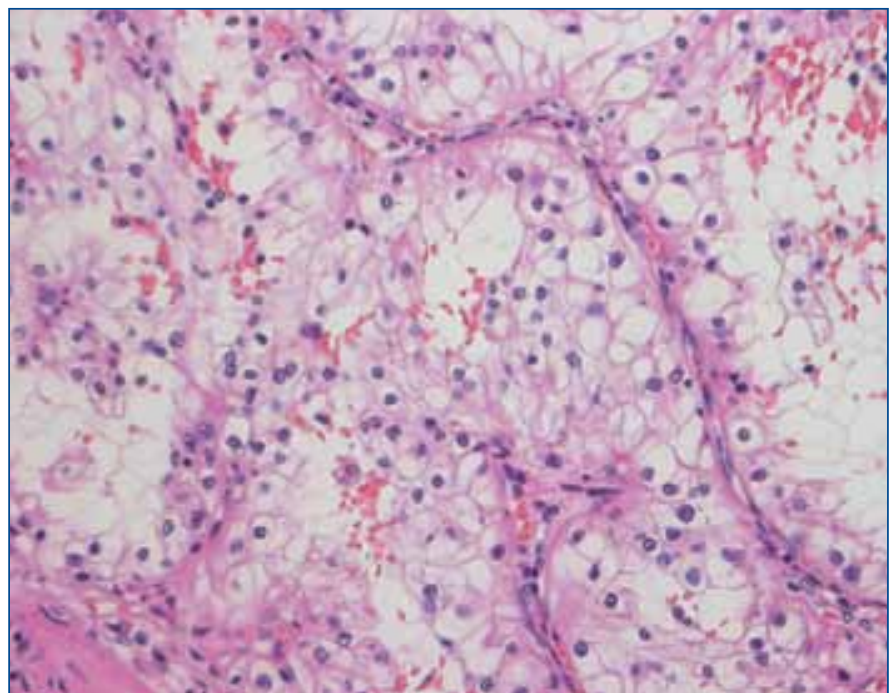

Fig. 2. Hematoxylin and eosin staining demonstrating tumour cells with an alveolar structure, clear cytoplasms, and small nucleoli showing characteristic histology of renal cell carcinoma (magnification $\times 400$ ). 
Jeong et al.

\section{References}

1. Dursun H, Bayazit AK, Buyukcelik M, et al. Associated anomalies in children with congenital solitary functioning kidney. Pediatr Surg Int 2005;21:456-9. http://dx.doi.org/10.1007/s00383-005-1408-7

2. Yalavarth R, Parikh CR. Congenital renal agenesis: A review. Saudi J Kidney Dis Transpl 2003;14:336-41.

3. Woolf AS, Hillman KA. Unilateral renal agenesis and the congenital solitary functioning kidney: Developmental, genetic and clinical perspectives. BJU Int 2007;99:17-21. http://dx.doi.org/10.1111/ j.1464-410X.2006.06504.x

4. Tripp BM, Homsy YL. Neonatal hydronephrosis - the controversy and the management. Pediatr Nephrol 1995;9:503-9. http://dx.doi.org/10.1007/BF00866741

5. Schuessler WW, Grune MT, Tecuanhuey LV, et al. Laparoscopic dismembered pyeloplasty. J Urol 1993;150:1795-9
6. Abraham GP, Siddaiah AT, Ramaswami K, et al. Laparoscopic management of recurrent ureteropelvic junction obstruction following pyeloplasty. Urol Ann 2015;7:183-7. http://dx.doi.org/10.4103/09747796.150489

7. Novak R, Muligan D, Abaza R. Robotic partial nephrectomy without renal ischemia. Urology 2012;79:1296301. http://dx.doi.org/10.1016/i.urology.2012.01.065

8. Nohara T, Fujita H, Yamamoto K, al. Modified anatropic partial nephrectomy with selective renal segmental artery clamping to preserve renal function: A preliminary report. Int J Urol 2008; 15:961-6. http://dx.doi. org/10.1111/i.1442-2042.2008.02141.x

Correspondence: Dr. Yu Seob Shin, Department of Urology, Chonbuk National University Medical School, and Research Institute of Clinical Medicine of Chonbuk National University-Biomedical Research Institute of Chonbuk National University Hospital, Jeonju, Korea; ball1210@hanmail.net 